\title{
Das ESR-Linienprofil antiferromagnetischer Verbindungen und die Abweichungen von der Lorentz-Form
}

\author{
K. Dräger \\ Institut für Physikalische Chemie, Universität Hamburg \\ Z. Naturforsch. 40 a, 251-259 (1985); received December 20, 1984
}

\begin{abstract}
The ESR-absorption of Antiferromagnetic Compounds and its Deviation from the Lorentzian Profile
The ESR-absorption of antiferromagnetic compounds is usually described by a Lorentzian profile given by $\left(1+z^{2}\right)^{-1}$ with $z=\left(H-H_{0}\right) / \Delta H$. To obtain further improvements an additional term of 4 th order is introduced. This modified profile given by $\left(1+z^{2}+\alpha z^{4}\right)^{-1}$ with $\alpha>0$ changes the properties of several ESR parameters. First of all there is a finite second moment which is given by $\alpha^{-1 / 4} \cdot \Delta H$. For small values of $\alpha$ the integrated intensity will be reduced by $\alpha^{1 / 2}$ while the differentiated line-width $\Delta H_{\mathrm{pp}}$ increases linearly with $\alpha$.

The application of this modified Lorentzian profile to the ESR spectra of antiferromagnetic $\mathrm{Cr}_{2} \mathrm{O}_{3}$ and $\mathrm{MnF}_{2}$ results in an improved fit. In both cases, the parameter $\alpha$ took on values between $(20-100) \cdot 10^{-4}$. For $\mathrm{Cr}_{2} \mathrm{O}_{3}$ there is an increase of $\alpha$ for rising temperature in the range from $320 \mathrm{~K}$ to $520 \mathrm{~K}$. The second moment of $\mathrm{Cr}_{2} \mathrm{O}_{3}$ at $T=330 \mathrm{~K}$ was found to be $2100 \mathrm{G}$. For $\mathrm{MnF}_{2}$ a value of $1225 \mathrm{G}$ was derived which is in rough accordance with results of Van Vleck's theory of line broadening.
\end{abstract}

\section{Einleitung}

Die Diskussion zur Linienform von ESR-Signalen, die an paramagnetischen Proben beobachtet werden, bewegt sich in der Regel in einem Rahmen, der durch die beiden Grenzformen des Lorentzund des Gaußprofils vorgezeichnet ist. Für beide Typen ist in der Literatur [1-5] eine größere Zahl von Beispielen bekannt, die die Bedeutung dieser Grundformen hinreichend belegen.

Insbesondere hat sich das Lorentzprofil vor allem bei Verbindungen bewährt, die durch eine antiferromagnetische Kopplung ihrer paramagnetischen Metall-Ionen gekennzeichnet sind. In diesen Systemen zerfällt oberhalb des Néel-Punktes $T_{\mathrm{N}}$ die zuvor stabile Ordnung der Spins, und die Probe wird ESR-aktiv. Im Experiment beobachtet man eine relativ breite Absorptionslinie, der schon recht früh die Nähe zum Lorentzprofil zugesprochen wurde $[2,6]$.

Später haben Mori und Kawasaki [7] dann theoretisch nachweisen können, daß bei antiferromagnetischen Verbindungen - und dort im Grenzfall hoher Temperatur - Resonanzlinien vom Lorentztyp in der Tat erwartet werden. Diese Vorhersage ist

Sonderdruckanforderungen an Dr. K. Dräger, Institut für Physikalische Chemie der Universität Hamburg, Laufgraben 24, D-2000 Hamburg 13, FRG. durch quantitative Untersuchungen an verschiedenen Systemen - so an $\mathrm{Cr}_{2} \mathrm{O}_{3}$ [8], $\mathrm{MnO}$ [9] und an $\mathrm{MnF}_{2}[10]$ - bestätigt worden. Dabei ist hervorzuheben, daß die beobachtete Linienform nicht erst im Grenzfall dem Lorentztyp entspricht, sondern bereits bei Temperaturen, die nur um $\Delta T=10 \mathrm{~K}$ über der Néel-Temperatur des jeweiligen Systems liegen.

Ungeachtet dieser guten Übereinstimmung ergeben sich jedoch, und zwar gerade für das LorentzProfil, hieraus Schwierigkeiten von grundsätzlicher Art. Diese werden bei dem Versuch sichtbar, die verschiedenen Ansätze, mit denen der Mechanismus der Linienverbreiterung untersucht worden ist, in ein widerspruchsfreies Verhältnis zu bringen.

Als Ausgangspunkt einer solchen Erörterung bietet sich die von Van Vleck [11] in die Theorie der magnetischen Resonanz eingeführte Methode der Momente an. Danach läßt sich für jeden ESR-aktiven Zustand, und zwar abhängig nur von den Konstanten des Spin-Hamilton-Operators, jeweils das 2. Moment einer Resonanzlinie mit dem Zentrum bei $H_{0}$ gemä $\beta\left\langle\left(H-H_{0}\right)^{2}\right\rangle$ bestimmen. Mit dieser Angabe verfügt man in jedem Fall über eine endliche Größe, die zu der im ESR-Experiment gemessenen Linienbreite in unmittelbarem Zusammenhang steht.

Diesem Sachverhalt auf der einen Seite steht nun das Ergebnis auf der anderen gegenüber, daß das 2.

0340-4811 / 85 / 0300-0251 \$ 01.30/0. - Please order a reprint rather than making your own copy. 
Moment einer Resonanzlinie, die in ihrem Verlauf exakt der Lorentz-Form entspricht, generell über alle Grenzen wächst. Um dieses Defizit auszugleichen, wird in der vorliegenden Arbeit das Modell einer Linienform diskutiert - zunächst theoretisch und danach in seiner Anwendung auf zwei experimentelle Beispiele -, das als Erweiterung des idealen Lorentz-Profils anzusehen ist. Im Unterschied zu diesem besitzt es jedoch ein endliches 2. Moment. Es kann daher zu einer Verbesserung der Aussagen beitragen, die prinzipiell über gemessene Resonanzlinien zu erlangen sind.

\section{Das modifizierte Lorentz-Profil}

Resonanzlinien, die durch weitreichende Ausläufer in den Flanken des Absorptionssignals gekennzeichnet sind, lassen sich - soweit die Symmetrie des Signals sichergestellt ist - durch das LorentzProfil beschreiben. Mit nur einem Parameter $\Delta H$ ausgestattet, der den Feldbereich um das Zentrum $H_{0}$ charakterisiert, wo der wesentliche Teil der Absorption erfolgt, wird dieses Profil formal durch den Ausdruck

$$
l(H)=\frac{1}{1+\left(\frac{H-H_{0}}{\Delta H}\right)^{2}}
$$

dargestellt. Die mit (1) verbundene Gesamtintensität der Resonanz ergibt sich dann zu

$$
I(0)=N \int_{-\infty}^{-\infty} l(H) \mathrm{d} H,
$$

wobei $N$ eine für die Normierung des Profils erforderliche Konstante ist. Mit (1) und der Substitution

$$
z=\left(H-H_{0}\right) / \Delta H
$$

ergibt sich dann die Gesamtintensität

$$
I(0)=N \Delta H L(0) \text {. }
$$

Der Faktor $L(0)$ ist im Hinblick auf die vorgesehene Modifikation gewählt und erfaßt den Einfluß der Linienform zu

$$
L(0)=\int_{-\infty}^{+\infty} \frac{\mathrm{d} z}{1+z^{2}}=\pi
$$

Die für das Lorentz-Profil eingangs schon erwähnte Divergenz des 2. Moments folgt mit (2) und (3) aus der Definitionsgleichung

$$
\left\langle\left(H-H_{0}\right)^{2}\right\rangle I(0)=N(\Delta H)^{3} \int_{-\infty}^{+\infty} \frac{z^{2}}{1+z^{2}} \mathrm{~d} z .
$$

Denn für große Beträge von $z$ strebt der Integrand gegen den Wert 1. Die im folgenden näher untersuchte Modifikation des Lorentz-Profils, dargestellt durch den Ansatz

$$
l(z)=\frac{1}{1+z^{2}+\alpha z^{4}},
$$

vermeidet diese Schwierigkeit. Der Zusatzterm von 4. Ordnung in $z$ schließt eine Divergenz des 2. Moments aus. Darüber hinaus kann durch die Einschränkung $0<\alpha \ll 1$ noch erreicht werden, daß im Hauptgebiet der Resonanz $(z<1)$ die Übereinstimmung mit dem Lorentz-Profil weitgehend erhalten bleibt. Im Hinblick auf das Experiment verfügt man damit neben der Größe $\Delta H$ nach (3) noch über einen zweiten freien Parameter, durch den die Anpassung an eine gemessene Resonanzkurve gegebenenfalls weiter verbessert werden kann. Zunächst sollen aber die allgemeinen Folgen diskutiert werden, die mit der obigen Modifikation verbunden sind.

Unter den mit (7) geänderten Bedingungen ergibt sich zunächst die Gesamtintensität zu

$$
I(\alpha)=N \Delta H L(\alpha) .
$$

Der Einfluß des Parameters $\alpha$ auf die Intensität wird dabei durch das Integral beschrieben

$$
L(\alpha)=\int_{-\infty}^{-\infty} \frac{\mathrm{d} z}{1+z^{2}+\alpha z^{4}} .
$$

Mit dieser Kennzeichnung wird der Anschluß an (4) bzw. (5) hergestellt. Die weitere Auswertung setzt die Kenntnis der Polstellen $z_{\mu}$ von (7) in der komplexen Zahlenebene voraus. Aus der Forderung

$$
1+z_{\mu}^{2}+\alpha z_{\mu}^{4}=0
$$

sowie der Nebenbedingung $1-4 \alpha>0$, die die Nähe zum reinen Lorentz-Profil sichert, errechnet man

$$
\begin{aligned}
& z_{1}=\frac{i}{2 \alpha} \sqrt{1-\sqrt{1-4 \alpha}}, \\
& z_{2}=\frac{i}{2 \alpha} \sqrt{1+\sqrt{1-4 \alpha}}, \\
& z_{3}=-z_{1} \text { und } z_{4}=-z_{2} .
\end{aligned}
$$

Diese Ergebnisse erlauben eine Partialbruchzerlegung des Integranden von (9) gemäß

$$
l(z)=\sum_{\mu=1}^{4} \frac{l \mu}{z-z_{\mu}} .
$$


In der vorliegenden Form genügt die Funktion $l(z)$ den Bedingungen, die im Anhang ausführlicher erörtert worden sind. Vor allem erzeugt die Rückführung von (12) in die Ausgangsform (9) weitere Nebenbedingungen, die im Anschluß an (A4) durch

$$
g_{0}=1 / \alpha \text { und } g_{2}=0
$$

zu beschreiben sind. Für die Koeffizienten von (12) ergibt sich daraus unter Beachtung von (A 7)

$$
\begin{aligned}
& l_{1}=+\frac{1}{2 \alpha} \frac{1}{z_{1}} \frac{1}{z_{1}^{2}-z_{2}^{2}}, \\
& l_{2}=+\frac{1}{2 \alpha} \frac{1}{z_{2}} \frac{1}{z_{2}^{2}-z_{1}^{2}} .
\end{aligned}
$$

Diese Teilergebnisse bestimmen bereits im wesentlichen den Wert des Integrals (9). Da der Integrand alle Eigenschaften der im Anhang diskutierten Funktionsklasse besitzt, folgt mit (A 15) für das Integral sofort

$$
L(\alpha)=2 \pi \mathrm{i}\left(l_{1}+l_{2}\right) .
$$

Die Substitution der Koeffizienten gemäß (14) führt zusammen mit den Polstellen nach (10) auf das Resultat

$L(\alpha)=\pi\left[\frac{\sqrt{2}}{\sqrt{1+\sqrt{1-4 \alpha}}+\sqrt{1-\sqrt{1-4 \alpha}}}\right]$.

Für den Grenzfall $\alpha \rightarrow 0$ strebt der Ausdruck in der Klammer gegen den Wert 1 und erreicht den Wert des reinen Lorentz-Profils.

Einen Überblick vom Gesamtverhalten der relativen Größe $L(\alpha) / L(0)$ und somit ihres Einflusses auf die integrale Intensität der Absorption gibt Abbildung 1. Danach bewirkt ein Anwachsen der Größe $\alpha$ eine zunächst steile, sich dann aber verflachende Abnahme, die im Geltungsbereich $1-4 \alpha>0$ zu einer Reduktion der Intensität von fast 30\% führen kann. Der Abfall im Anfangsteil befolgt dabei ein Wurzelgesetz. Als Näherung für den Bereich $0 \leqq \alpha \ll 0.25$ findet man

$$
L(\alpha)=L(0)[1-\sqrt{\alpha}] .
$$

Damit ist gezeigt, daß Abweichungen vom LorentzProfil gemäß der nach (7) diskutierten Art, dort, wo sie tatsächlich auftreten, durchaus einen beachtenswerten Einfluß auf die integrale Intensität nehmen können. Dieser Sachverhalt gewinnt an Bedeutung, wenn man die Folgen bedenkt, die die besondere Nachweistechnik der magnetischen Resonanzspek- troskopie nach sich zieht. Im Experiment wird bekanntlich die 1. Ableitung des eigentlichen Absorptionssignals aufgezeichnet. Abbildung 2 zeigt dazu die realen Verhältnisse am Beispiel eines $\mathrm{Cr}_{2} \mathrm{O}_{3}$-Spektrums.

Als charakteristische Größen sind die Signalhöhe $S$, als Spitze/Spitze-Abstand, und die differentielle Linienbreite $\Delta H_{\mathrm{pp}}$ anzusehen. Im Falle eines reinen Lorentz-Profils reicht bereits deren Kenntnis aus, um die integrale Intensität gemä $\beta$

$$
I(0)=\frac{\pi}{\sqrt{3}} S\left[\Delta H_{\mathrm{pp}}\right]^{2}
$$

exakt zu bestimmen. Für das modifizierte LorentzProfil (7) ist eine entsprechende Relation nicht mehr direkt sondern nur mit Hilfe eines Korrekturfaktors $K_{\mathrm{f}}$ herzustellen, der dem Einfluß von $\alpha$

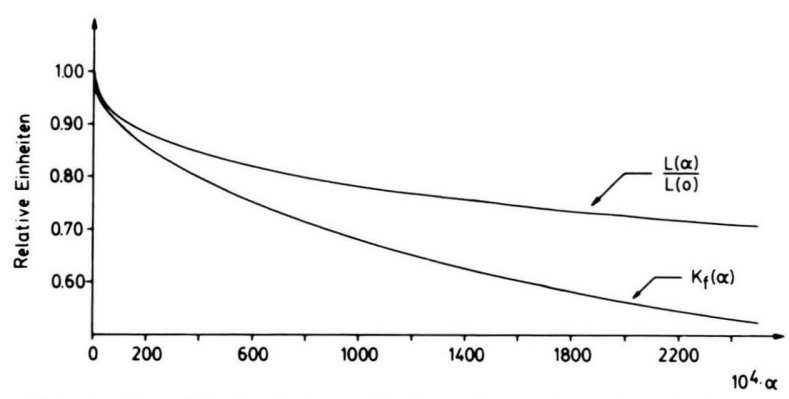

Abb. 1. Der Verlauf der relativen Intensität, beschrieben durch den Quotienten $L(\alpha) / L(0)$, in Abhängigkeit von $\alpha$ und der entsprechende Verlauf des Korrekturfaktors $K_{f}(\alpha)$ nach (19), wenn die integrale Intensität aus den charakteristischen Daten des differenzierten Signals bestimmt wird.

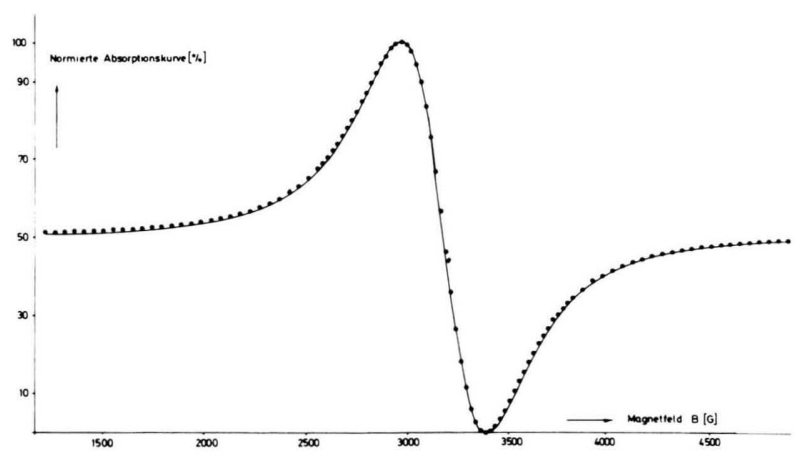

Abb. 2. Die normierte ESR-Absorption einer polykristallinen $\mathrm{Cr}_{2} \mathrm{O}_{3}$-Probe, dargestellt durch Meßpunkte zur 1 . Ableitung des Signals. Der Kurvenzug ist Ergebnis einer Anpassung gemäß (33). 
Rechnung trägt. In Analogie zu (18) empfiehlt sich für die integrale Intensität die Darstellung

$$
I(\alpha)=K_{\mathrm{f}}(\alpha) \frac{\pi}{\sqrt{3}} S(\alpha)\left[\Delta H_{\mathrm{pp}}(\alpha)\right]^{2} .
$$

Sie normiert den Übergang zum reinen LorentzProfil durch $K_{\mathrm{f}}(0)=1$. Der weitere Verlauf von $K_{\mathrm{f}}(\alpha)$ ergibt sich mit (8) und (16) im Rahmen einer numerischen Auswertung. Er ist in Abb. 1 und dort durch die 2. Kurve graphisch dargestellt worden.

Hier zeigt sich, daß eine unkritische Verwendung von Signalhöhe $S$ und differentieller Linienbreite $\Delta H_{\mathrm{pp}}$ bei der Bestimmung der Intensität nach (18) zu ganz beträchtlichen Fehleinschätzungen führt. Abweichungen bis zu 50\% im Geltungsbereich von $\alpha$ können auftreten; selbst für Werte um $\alpha=0.01$ sind noch Fehler von 10\% zu erwarten. Diesem Sachverhalt liegt die unterschiedliche Wirkung des Linienformfaktors $\alpha$ auf die integrale Intensität einerseits und auf die charakteristischen Größen $S$ und $\Delta H_{\mathrm{pp}}$ andererseits zugrunde.

Von diesen sei hier die Linienbreite $\Delta H_{\mathrm{pp}}$ nochmals hervorgehoben, da sie - vor allem experimentell - eine gut überprüfbare Größe darstellt. Dabei ist sie deutlich gegen die Halbwertsbreite

$$
\Delta H_{1 / 2}=H_{2}-H_{1}
$$

abzusetzen, deren Feldwerte $H_{\mathrm{i}}$ aus dem Intensitätsvergleich

$$
I\left(H_{\mathrm{i}}\right)=\frac{1}{2} I\left(H_{0}\right) \quad \text { mit } \quad i=1,2
$$

an der ursprünglichen Absorptionskurve stammen. Ein unterschiedliches Verhalten ist hier zu beachten. Beim modifizierten Lorentz-Profil ergibt sich diese Größe, die Halbwertsbreite, explizit zu

$$
\Delta H_{1 / 2}=2 \cdot \sqrt{\frac{2}{1+\sqrt{4 \alpha+1}}} \Delta H .
$$

Sie nimmt danach mit wachsendem $\alpha$ ab. Für die differentielle Linienbreite $\Delta H_{\mathrm{pp}}$, die bei der im ESR-Experiment üblichen Nachweistechnik auftritt, ist außer für $\alpha=0$ mit $\Delta H_{\mathrm{pp}}(0)=2 / \sqrt{3} \cdot \Delta H$ kein geschlossener Ausdruck zu erreichen. $\Delta H_{\mathrm{pp}}(\alpha)$ ergibt sich aus den Lösungen der Gleichung

$$
1+3[2 \alpha-1] z_{0}^{2}-9 \alpha z_{0}^{4}-10 \alpha^{2} z_{0}^{6}=0,
$$

die in dieser Form die Lage der Wendepunkte für das Profil (7) beschreibt.

Abbildung 3 faßt die Ergebnisse der numerischen Auswertung von (20) durch einen Koeffizienten $\varrho(\alpha)$ zusammen, der die Abhängigkeit der Linienbreite von $\alpha$ in folgender Weise definiert:

$$
\Delta H_{\mathrm{pp}}(\alpha)=\varrho(\alpha) \Delta H_{\mathrm{pp}}(0) .
$$

Danach nimmt die differentielle Linienbreite mit wachsendem $\alpha$ gleichmäßig zu. Allerdings bleibt der Anstieg unter 9\% des Ausgangswertes. Bei dieser Sachlage kann der letzte Summand in (20) als klein gegenüber den anderen eingestuft werden. Der Einfluß von $\alpha$ auf die differentielle Linienbreite ergibt sich dann explizit - wenn auch nur näherungsweise - durch

$$
\Delta H_{\mathrm{pp}}(\alpha)=\left[1+\frac{1}{2} \alpha\right] \Delta H_{\mathrm{pp}}(0) .
$$

Mit diesen Ausführungen ist gezeigt worden, daß selbst unter der einschränkenden Bedingung $\alpha \ll 1$ der Linienformfaktor einen zwar begrenzten, im Rahmen der heute verfügbaren Meßgenauigkeit aber durchaus nennenswerten Einfluß auf die beiden Resonanzparamter Gesamtintensität und differentielle Linienbreite nehmen kann.

Die Diskussion hat bislang einen Aspekt für die Einführung des Linienformparamters $\alpha$ aufzeigen können. Es gibt weitere. Ein sogar ungleich größeres Gewicht besitzt $\alpha$ bei der Berechnung des 2. Momentes der Resonanz. In Anlehnung an (6) gilt für das modifizierte Lorentz-Profil

$$
\left\langle\left(H-H_{0}\right)^{2}\right\rangle I(\alpha)=N(\Delta H)^{3} M(\alpha)
$$

mit der Abkürzung

$$
M(\alpha)=\int_{-\infty}^{+\infty} \frac{z^{2}}{1+z^{2}+\alpha z^{4}} \mathrm{~d} z
$$

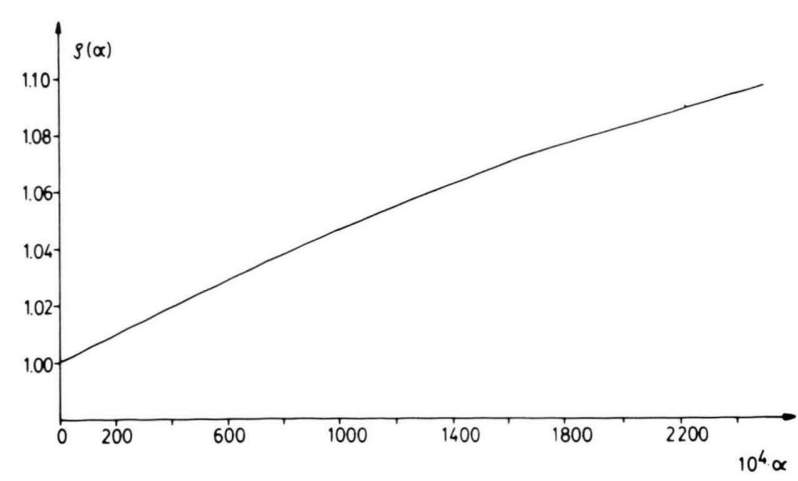

Abb. 3. Die relative Zunahme $\varrho(\alpha)$ nach (21) der differentiellen Linienbreite $\Delta H_{\mathrm{pp}}$ in Abhängigkeit vom Parameter $\alpha$ des modifizierten Lorentz-Profils. 
Die Auswertung dieses Ausdrucks erfolgt analog zu früherem. Den Integranden von (24) bezeichnen wir mit $m(z)$. Seine Pole stimmen ersichtlich mit denen des modifizierten Lorentz-Profil überein, so da $\beta$ von einer Partialbruchzerlegung der Form

$$
m(z)=\sum_{\mu=1}^{4} \frac{m_{\mu}}{z-z_{\mu}}
$$

ausgegangen werden kann. Die Überführung von (25) in die äquivalente Darstellung (A3) gelingt dabei hinsichtlich der Koeffizienten durch die Forderung

$$
g_{0}=0 \text { und } g_{2}=1 / \alpha .
$$

Aus diesen Nebenbedingungen sowie den Relationen (A4.1) bzw. (A4.3) folgt dann für die Koeffizienten der Darstellung (25)

$$
\begin{aligned}
& m_{1}=\frac{1}{2 \alpha} \frac{z_{1}}{z_{1}^{2}-z_{2}^{2}}, \\
& m_{2}=\frac{1}{2 \alpha} \frac{z_{2}}{z_{2}^{2}-z_{1}^{2}},
\end{aligned}
$$

während $m_{3}$ und $m_{4}$ wiederum durch (A 7) festgelegt sind. Die Bestimmung des 2. Momentes gelingt in zwei Schritten. Zunächst folgt mit (A15) für das Integral (24)

$$
M(\alpha)=2 \pi i\left(m_{1}+m_{2}\right) .
$$

Mit diesem sowie dem entsprechenden Resultat (15) für $I(\alpha)$ ergibt sich danach

$$
\left\langle\left(H-H_{0}\right)^{2}\right\rangle=(\Delta H)^{2} \frac{m_{1}+m_{2}}{l_{1}+l_{2}} .
$$

Der so erhaltene Ausdruck läßt sich durch Einsetzen der Koeffizienten gemäß (14) bzw. (27) weiter vereinfachen; man erhält

$$
\left\langle\left(H-H_{0}\right)^{2}\right\rangle=\frac{(\Delta H)^{2}}{\sqrt{\alpha}} .
$$

Dieses - in seiner Form einfache - Ergebnis macht den Stellenwert des Parameters $\alpha$ besonders deutlich und legt den Versuch nahe, bei Lorentz-ähnlichen Resonanzlinien eine zweiparametrige Beschreibung mit (7) vorzunehmen. Bereits ein Wert von $\alpha=10^{-4}$ - der bei der den nachfolgend beschriebenen Experimenten nicht unterschritten wird - führt auf eine akzeptable Größenordnung für das 2. Moment. Am einfachsten ist dies durch einen Vergleich mit der differentiellen Linienbreite $\Delta H_{\mathrm{pp}}(\alpha)$ nachzuweisen. Mit Hilfe von (21) überführt man dazu (30) in die Form

$$
\sqrt{\left\langle\left(H-H_{0}\right)^{2}\right\rangle}=\left[\frac{\sqrt{3}}{2 \alpha^{1 / 4} \varrho(\alpha)}\right] \Delta H_{\mathrm{pp}}(\alpha)
$$

und schätzt den Wert der Klammer ab. Für $\alpha=10^{-4}$ errechnet man 8,66 mit der Tendenz, daß für größere Werte von $\alpha$ der Vorfaktor weiter abnimmt. Das nach (30) bestimmte 2. Moment steht unter diesen Voraussetzungen in einer vernünftigen Relation zur differentiellen Linienbreite.

Im Ergebnis ist damit festzuhalten, daß das modifizierte Lorentz-Profil (7) einen aussichtsreichen Ansatz bietet, um experimentelle Resonanzlinien durch eine unabhängige Variation in den Parametern $\Delta H$ und $\alpha$ formgetreu zu beschreiben. Darüber hinaus stellt das dabei erzielbare 2. Moment eine zusätzliche Information über den Gesamtvorgang der Resonanzabsorption dar.

\section{Experimentelle Überprifung}

Die Frage, inwieweit das modifizierte Linienprofil (7) geeignet ist, um die gemessenen ESR-Spektren antiferromagnetischer Verbindungen in verbesserter Weise $\mathrm{zu}$ beschreiben, haben wir an den klassischen Beispielen $\alpha-\mathrm{Cr}_{2} \mathrm{O}_{3}$ und $\mathrm{MnF}_{2}$ untersucht. Die Untersuchung wurde an einem X-BandSpektrometer (Bruker) im Mikrowellenbereich von 9,3 GHz durchgeführt und war so angelegt, daß die Messungen auch bei unterschiedlichen Temperaturen wiederholt werden konnten. Die Stabilität des gesamten Meßaufbaus stellt dabei eine wesentliche Voraussetzung dar, um den Parameter $\alpha$ und seinen Temperaturgang $\mathrm{zu}$ bestimmen. Die apparativen Anforderungen hinsichtlich des Parameters $\Delta H$ sind demgegenüber geringer anzusetzen und im Regelfall erfüllt.

Für die hier betrachteten polykristallinen Verbindungen ist vom Autor $[8,10]$ schon früher gezeigt worden, daß ihre ESR-Absorption weitgehend Lorentz-Charakter hat. Das dort vorgeschlagene Variationsverfahren wird auch im folgenden angewandt. Es nimmt, wie schon erwähnt, Bezug auf die 1. Ableitung der Resonanzkurve, da das Meßsignal zunächst nur in dieser Form zur Verfügung steht. 
Diese Vorgabe macht im weiteren den Übergang von der Funktion $l(H)$ gemäß (3) und (7) zur Ableitung

$$
\begin{aligned}
l^{\prime}(H)=- & \frac{2 N}{\Delta H}\left(\frac{H-H_{0}}{\Delta H}\right) \\
& \frac{1+2 \alpha\left(\frac{H-H_{0}}{\Delta H}\right)^{2}}{\left[1+\left(\frac{H-H_{0}}{\Delta H}\right)^{2}+\alpha\left(\frac{H-H_{0}}{\Delta H}\right)^{4}\right]^{2}}
\end{aligned}
$$

erforderlich. Darüber hinaus bedarf es noch der Vereinbarung, daß der Spitze/Spitze-Abstand des gemessenen Signals wie auch jener des theoretischen Profils jeweils auf den Wert 1 normiert wird.

In dem so festgelegten Rahmen erfolgt die eigentliche Anpassung des theoretischen Profils mit einem Raster von $N$ Bezugspunkten $\left(H_{i}, Y_{i}^{\prime}\right)$, die der kontinuierlich aufgezeichneten Resonanzkurve entnommen werden. Die Entscheidung der Frage, wann ein gegebenes Parameterpaar $(\Delta H, \alpha)$ die Resonanzkurve optimal beschreibt, wird dabei von dem Verhalten der Fehlerfunktion

$$
S(\Delta H, \alpha)=\sqrt{\frac{1}{N} \sum_{i=1}^{N}\left[Y_{i}^{\prime}-1^{\prime}\left(H_{i}\right)\right]^{2}}
$$

abhängig gemacht. Von ihrer Konstruktion her stellt (33) den mittleren Fehler über den Bereich der Kurvenauswertung dar. Die Konkurrenzlage zum reinen Lorentz-Profil, um die es geht, ergibt sich dabei aus der folgenden Einschätzung. Ein Parameterpaar $(\Delta H, \alpha)$ mit $\alpha>0$, das die Fehlerfunktion $S$ in einem Minimum beschreibt und im übrigen für beliebige Werte $\Delta H^{*}$ der Bedingung

$$
S(\Delta H, \alpha)<S\left(\Delta H^{*}, 0\right)
$$

genügt, verbessert in jedem Fall die Anpassung auf einen vorgegebenen experimentellen Verlauf und zeichnet damit das modifizierte Profil (7) gegenüber dem Standard-Typ aus.

Mit diesem Ansatz ergeben die Messungen an $\alpha-\mathrm{Cr}_{2} \mathrm{O}_{3}$, daß das modifizierte Profil in jedem Fall, d. h. hier bei allen Temperaturen der Untersuchung, eine verbesserte Beschreibung erlaubt. Die Resultate der mit jeweils 80 experimentellen Wertepaaren durchgeführten Optimierung sind in Abb. 4 graphisch dargestellt worden. Die Abnahme von $\Delta H$ mit steigender Temperatur entspricht dabei dem bekannten Verhalten der differentiellen Linienbreite [12].
Der Anstieg von $\alpha$ mit wachsender Temperatur ist dagegen sehr erstaunlich, insofern als die Theorie [7] gerade für steigende Temperaturen das Gegenteil, nämlich eine Abnahme und damit die Tendenz zum reinen Lorentz-Profil favorisiert. Diese Beobachtung läßt vermuten, daß beim $\mathrm{Cr}_{2} \mathrm{O}_{3}-$ möglicherweise strukturbedingt - eine magnetische Kopplung wirksam wird, die bei der HochtemperaturNäherung, wie sie Mori und Kawasaki [7] angegeben haben, nicht ohne weiteres zu berücksichtigen ist.

Eine solche Verkürzung wird unter den von Van Vleck [11] gewählten Annahmen, für die er eine Linienverbreiterung beschreibt, vermieden. Das durch ihn eingeführte 2. Moment der magnetischen Resonanz hat in jedem Fall einen endlichen Wert. Im Hinblick auf diese Größe stellt man für $\mathrm{Cr}_{2} \mathrm{O}_{3}$ fest, daß das durch Optimierung gewonnene 2 . Moment im untersuchten Temperaturbereich abnimmt. Die Größenordnung sei an zwei Beispielen verdeutlicht. Bei der Temperatur $T=325 \mathrm{~K}$, d. h. etwa $20 \mathrm{~K}$ oberhalb der Néel-Temperatur, findet man für das 2. Moment $\sqrt{\left\langle\left(H-H_{0}\right)^{2}\right\rangle}=2210 \mathrm{G}$; dagegen wird für $T=513 \mathrm{~K}$ ein Feldwert von $990 \mathrm{G}$ ermittelt. Beide Werte liegen oberhalb der differentiellen Linienbreite. Ansonsten scheint die Abnahme - wie bereits angedeutet - in erster Linie die spezifischen Eigenschaften der Verbindung widerzuspiegeln.

Diese Wertung ergibt sich auch aus einem Vergleich mit den an $\mathrm{MnF}_{2}$ gewonnenen Ergebnissen.

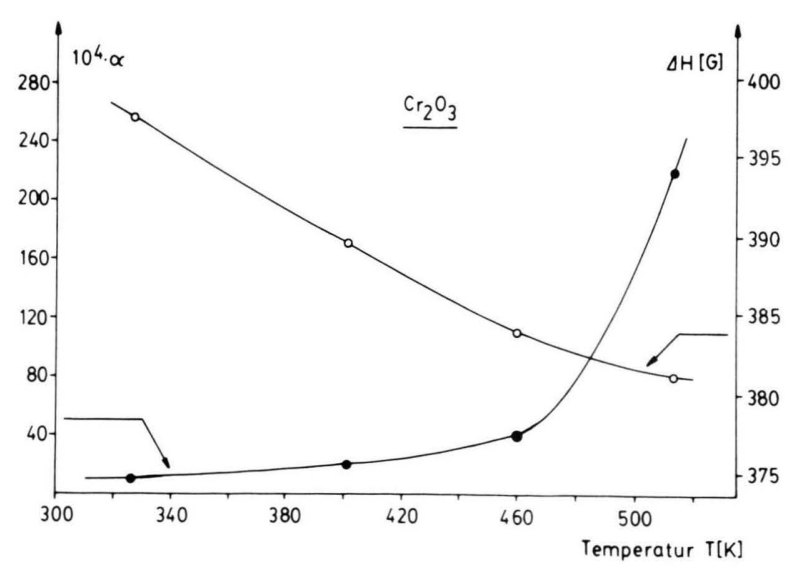

Abb. 4. Der Temperaturverlauf des Parameterpaares $(\Delta H, \alpha)$ für polykristallines $\mathrm{Cr}_{2} \mathrm{O}_{3}$ nach Anpassung des modifizierten Lorentz-Profils auf die zugehörigen gemessenen ESRSpektren. 


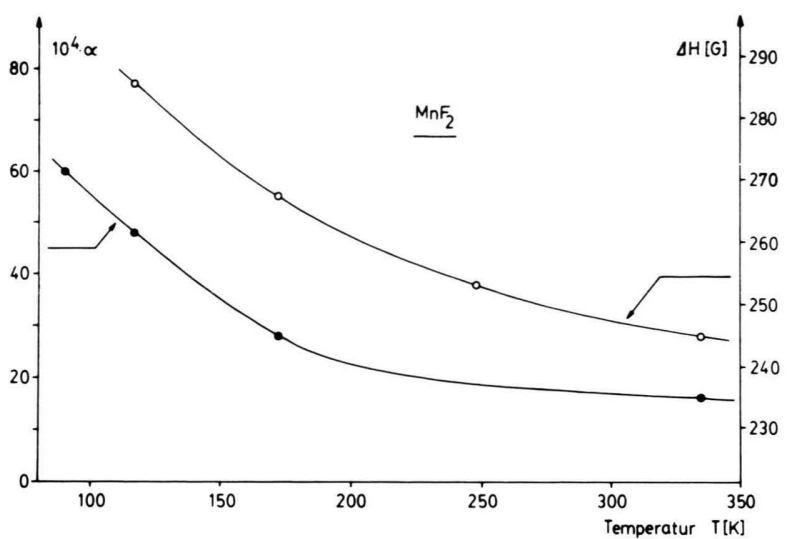

Abb. 5. Der Temperaturverlauf des Parameterpaares $(\Delta H, \alpha)$ für polykristallines $\mathrm{MnF}_{2}$ nach Anpassung des modifizierten Lorentz-Profils auf die zugehörigen gemessenen ESRSpektren.

Abbildung 5 zeigt zunächst den für diese Verbindung ermittelten Temperaturgang für die Parameter $\Delta H$ und $\alpha$. Wie schon zuvor sind jeweils 80 Wertepaare für die Optimierung verwendet worden.

Zunächst erweist sich auch hier die modifizierte Linienform (7) als dem Experiment angemessen, da die Extremalbedingung (34) für Werte mit $\alpha>0$ erfüllt werden kann. Gegenüber $\mathrm{Cr}_{2} \mathrm{O}_{3}$ zeigen die Parameter eine fallende Tendenz für steigende Temperaturen. Hinsichtlich des Parameters $\alpha$ erfüllt $\mathrm{MnF}_{2}$ damit die theoretischen Erwartungen [7] zum Linienprofil. Als Folge - und hier liegt der Unterschied zum vorigen Beispiel - beobachtet man ein anderes Verhalten beim 2. Moment der Resonanz.

Für $\sqrt{\left\langle\left(H-H_{0}\right)^{2}\right\rangle}$ findet man bei $T=117 \mathrm{~K}$ einen Feldwert von 1082 Gauss, der im weiteren noch steigt und bei $T=335 \mathrm{~K}$ einen Wert von 1225 Gauss erreicht. Das 2. Moment nimmt also in begrenztem Umfang zu.

Bei dieser Sachlage bietet es sich an, einen direkten Vergleich mit den Ergebnissen zu suchen, die aus der Van Vleckschen Theorie [11] abzuleiten sind. Das 2. Moment eines Systems, das - wie hier - im polykristallinen Zustand vorliegt und durch die Dipol-Dipol-Wechselwirkung identischer Spins der Quantenzahl $S$ erzeugt wird, beträgt danach

$\left\langle\left(H-H_{0}\right)^{2}\right\rangle=\frac{3}{5} g^{2} \mu_{\mathrm{B}}^{2} S(S+1) \sum_{i}^{\prime} \frac{1}{\boldsymbol{r}_{i}-\left.\boldsymbol{r}_{j}\right|^{6}}$.

Die Gittersumme erfaßt nur die Positionen $\boldsymbol{r}_{i}$ von paramagnetischen Ionen. Für das im Rutil-Typ kristallisierende $\mathrm{MnF}_{2}$ sind daher lediglich die Be- setzungen auf den Ecken und im Zentrum der Elementarzelle zu beachten. Etwa $10^{4}$ dieser Positionen reichen aus, um bei der Gittersumme Konvergenz zu erzeugen.

Mit den Abmessungen $a_{0}=48.73 \mathrm{~nm}, c_{0}=33.10 \mathrm{~nm}$ [13] sowie den magnetischen Daten $g=2.00$ und $S=5 / 2$ ergibt sich für $\mathrm{MnF}_{2}$ dann $\sqrt{\left\langle\left(H-H_{0}\right)^{2}\right\rangle}=$ $3032 \mathrm{G}$ als mittlere Abweichung der Resonanz.

Der Vergleich zeigt, daß die durch Messung und Linienformanalyse ermittelten 2. Momente von der gleichen Größenordnung sind. Im Detail fallen sie um einen Faktor 2,5 bis 2,8 kleiner aus als der Wert, den die Theorie nach (35) liefert. Diese Relation läßt vermuten, daß dem theoretischen Ergebnis die Bedeutung eines Grenzwertes für $T \rightarrow \infty$ zukommt.

Eine solche Wertung wird durch die Arbeiten von Anderson und Weiss [14, 15] gestützt. Die Autoren untersuchten den Einfluß der Austausch-Wechselwirkung auf die Breite der Resonanz und stellten im Ergebnis fest, daß - bei endlichen Temperaturen ein der Dipolverbreiterung gegenläufiger Effekt wirksam ist. Die von ihnen postulierte AustauschVerschmälerung reduziert die Linienbreite und führt so auch zu einer Abnahme des 2. Moments. In den Rahmen dieser inzwischen als gesichert geltenden Theorie vom Resonanzvorgang lassen sich die von uns aus der tatsächlichen Linienform ermittelten, kleineren 2. Momente zwanglos einfügen.

\section{Schlußbetrachtung}

In der vorliegenden Untersuchung ist an exemplarischen, antiferromagnetischen Verbindungen gezeigt worden, daß ihre ESR-Absorptionslinien mit einem modifizierten Lorentz-Profil gut zur Deckung gebracht werden können. Die mittlere Abweichung - genommen über den Gesamtbereich der Resonanz - liegt für alle Temperaturen zwischen $0.3 \%$ und $0.6 \%$. Gegenüber dem reinen Lorentz-Profil wurde nicht nur eine verbesserte Anpassung, sondern darüber hinaus eine quantitative Aussage zum 2. Moment der Resonanz erreicht, die zum Vergleich mit den Ergebnissen der Van Vleckschen Theorie herangezogen werden kann. Angaben zu höheren Momenten müssen dafür allerdings entfallen. Nach Grant [16] ist dieser Verzicht jedoch weniger schwerwiegend, da die höheren Momente und die Linienform nicht sehr eng aufeinander bezogen sind.

In dieser Beschränkung unterscheidet sich der hier unternommene Versuch sehr deutlich von an- 
deren, über die vor allem Poole [5] ausführlich berichtet. Die dort vorgestellten numerischen Verfahren zur Bestimmung der 2. und 4. Momente sind durch die Eigenart gekennzeichnet, daß die Festlegung des Feldbereichs, in dem die Resonanzkurve nach Bewertung des jeweiligen Experimentators aus dem Rauschuntergrund hervortritt, sehr stark auf das Ergebnis einwirkt. Nach Buluggi et al. [17] können Fehler von $50 \%$ bereits für das 2 . Moment der Resonanz auftreten.

Diese Unsicherheit kann in der vorliegenden Arbeit durch die Vorgabe eines Profils mit unbegrenztem Geltungsbereich und durch eine allgemein formulierte Extremalbedingung (34) vermieden werden. Dabei gilt der jeweilige mittlere Fehler der Anpassung zudem als wichtiger Indikator, durch den die Frage, inwieweit das Profil (7) zur Beschreibung eines resonanzfähigen Systems noch geeignet ist, stets neu aufgeworfen wird. Als Alternativen zum modifizierten Lorentz-Profil sind dabei die von Gulley et al. [18] formulierten, mehrparametrigen Profile zu betrachten, die zum Teil mit „Gauß-Faktoren“, zum Teil aber auch mit „Abschneidefeldern" definiert und von den Autoren im Hinblick auf die Verhältnisse der höheren Momente diskutiert worden sind. Solche Aspekte können - wie erwähnt - im Rahmen dieser Untersuchung nicht behandelt werden. Der Vorteil des hier vorgestellten, modifizierten Lorentz-Profils ist dafür seine Nähe zum theoretisch vorhergesagten Resonanzverhalten [7] und die relativ einfache Handhabung von Formeln für den Experimentator, der das Temperaturverhalten von Linienbreite, Intensität und 2. Moment einer geeigneten resonanzfähigen Verbindung untersuchen möchte.

\section{Anhang}

Die mathematische Behandlung des modifizierten Lorentz-Profils legt es nahe, die Eigenschaften spezieller komplexer Funktionen vom Typ

$$
f(z)=\sum_{\mu=1}^{4} \frac{f_{\mu}}{z-z_{\mu}}
$$

eingehender zu diskutieren. Die Pole $z_{\mu}$ der obigen Funktionen sind dabei so zu wählen, daß sie mit jenen des modifizierten Lorentz-Profils gemäß (10) und (11) übereinstimmen. Von diesen Ergebnissen berücksichtigen wir zunächst nur

$$
z_{3}=-z_{1} \quad \text { bzw. } z_{4}=-z_{2} .
$$

Bereits diese Festlegung bewirkt für die Koeffizienten $g_{v}$ der zu (A 1) äquivalenten Darstellung

$$
f(z)=\sum_{v=0}^{3} g_{v} z^{v} / \prod_{\mu=1}^{4}\left(z-z_{\mu}\right)
$$

eine erhebliche Vereinfachung. Unter Beachtung von (A2) findet man für die $g_{v}$ das folgende Gleichungssystem:

$$
\begin{aligned}
& g_{0}=-\left(f_{1}-f_{3}\right) z_{1} z_{2}^{2}-\left(f_{2}-f_{4}\right) z_{1}^{2} z_{2}, \\
& g_{1}=-\left(f_{1}+f_{3}\right) z_{2}^{2} \quad-\left(f_{2}+f_{4}\right) z_{1}^{2}, \\
& g_{2}=+\left(f_{1}-f_{3}\right) z_{1} \quad+\left(f_{2}-f_{4}\right) z_{2}, \\
& g_{3}=+\left(f_{1}+f_{3}\right) \quad+\left(f_{2}+f_{4}\right) .
\end{aligned}
$$

Das begrenzte Ziel, mit Hilfe von komplexen Funktionen Integrale des Typs

$$
\int_{-\infty}^{+\infty} \alpha \frac{g_{0}+g_{2} z^{2}}{1+z^{2}+\alpha z^{4}} \mathrm{~d} z
$$

zu berechnen, schränkt auch die Koeffizienten $f_{\mu}$ ein. So folgt aus den Bedingungen

$$
g_{1}=0 \text { und } g_{3}=0
$$

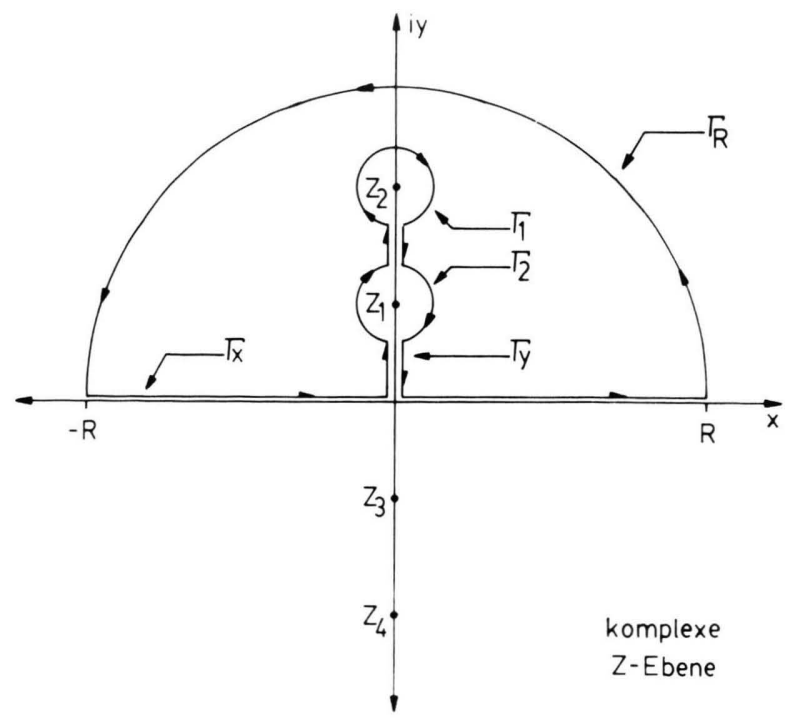

Abb. 6. Darstellung der Pole $z_{\mu}$ für das modifizierte Lorentz-Profil und Angabe eines geschlossenen Weges $\Gamma$, für den das Kurvenintegral (A 10) verschwindet. 
die von (A 5) ausgehen, für die Koeffizienten der Funktion $f(z)$ nach (A4) das Verknüpfungsschema

$$
f_{3}=-f_{1} \text { und } f_{4}=-f_{2} \text {. }
$$

Der nächste Schritt besteht in der genauen Beschreibung eines Gebietes $G$, in dem die Funktion $f(z)$ analytisch, d.h. ohne singuläres Verhalten ist. Für jede geschlossenen Kurve $\Gamma$, die ganz im Gebiet $G$ verläuft, gilt dann bekanntlich [19]:

$$
\oint_{\Gamma} f(z) \mathrm{d} z=0 .
$$

Um die Voraussetzungen zu erfüllen, definiert man für die durch (A 1) festgelegte Funktion $f(z)$ die folgenden Teilstrecken:

$$
\begin{aligned}
\Gamma_{x}: & -R \leqq z \leqq+R, \\
\Gamma_{j}: & z=z_{j}-i r e^{-i \varphi} \text { für } j=1,2 \\
& \text { mit } r \ll R \text { und } 0 \leqq \varphi \leqq 2 \pi, \\
\Gamma_{R}: & z=R e^{i \varphi} \\
& \text { mit }\left|z_{2}\right| \ll R \text { und } 0 \leqq \varphi \leqq \pi .
\end{aligned}
$$

Aus diesen Teilstrecken sowie zusätzlichen, geeigneten Abschnitten $\Gamma_{y}$ auf der imaginären Achse läßt sich ein geschlossener Kurvenzug

$$
\Gamma=\Gamma_{x}+\Gamma_{y}+\Gamma_{1}+\Gamma_{2}+\Gamma_{R}
$$

konstruieren, der ein Gebiet $G$ mit den geforderten Eigenschaften umschließt. Abbildung 6 gibt einen Überblick vom Verlauf des geschlossenen Weges $\Gamma$ und über die Lage der Pole der Funktion $f(z)$. Mit (A 1) folgt aus (A8) zunächst

$$
\sum_{\mu=1}^{4} f_{\mu} \oint_{\Gamma} \frac{\mathrm{d} z}{z-z_{\mu}}=0 .
$$

[1] B. Bleaney, Proc. Roy. Soc. London A 204, 203 (1950).

[2] H. Kumagai, K. Ono, I. Hayashi, H. Abe, H. Shono, H. Ibamoto u. S. Shimoda, Phys. Rev: 83, 1077 (1951); 85, 925 (1952).

[3] C. McLean u. G. J. Kor, Appl. Sci. Rev. B 4, 425 (1955).

[4] B. N. Misra u. S. K. Gupta, J. Mag. Res. 12, 126 (1973).

[5] C. Poole Jr., Electron Spin Resonance, Interscience Publishers, 1967

[6] E. P. Trouson, D. F. Bleil, R. K. Wangsness u. L. R. Maxwell, Phys. Rev. 79, 542 (1950).

[7] H. Mori u. K. Kawasaki, Progr. Theor. Phys. 28, 971 (1962).

[8] K. Dräger, Ber. Bunsenges. Phys. Chem. 79, 996 (1975).

[9] K. Dräger u. R. Kratz, Z. Naturforsch. 31 a, 1630 (1976).
Für den Fortgang ist es wichtig, die Beiträge der Teilstrecken - soweit sie sich nicht herausheben gesondert zu betrachten; im einzelnen sind das

$\Gamma_{x}: F_{R}(\alpha)=\sum_{\mu=1}^{4} f_{\mu} \int_{\Gamma_{x}} \frac{\mathrm{d} z}{z-z_{\mu}}$

$\Gamma_{j}: \oint_{\Gamma_{i}} \frac{\mathrm{d} z}{z-z_{\mu}}=2 \pi i \delta_{\mu j} \quad$ für $\quad j=1,2 ; \mu=1,2,3,4$

$\Gamma_{R}: \int_{\Gamma_{R}} \frac{\mathrm{d} z}{z-z_{\mu}}=i \pi+\ln \left(\frac{R+z_{\mu}}{R-z_{\mu}}\right)$.

Unter Beachtung der Nebenbedingung (A7) folgt aus (A 10) zunächst

$F_{R}(\alpha)-2 \pi i\left(f_{1}+f_{2}\right)+\sum_{\mu=1}^{4} f_{\mu} \ln \left(\frac{R+z_{\mu}}{R-z_{\mu}}\right)=0$.

Für den hier interessierenden Übergang $R \rightarrow \infty$ mit

$$
F(\alpha)=\lim _{R \rightarrow \infty} F_{R}(\alpha)
$$

vereinfacht sich wegen

$$
\lim _{R \rightarrow \infty} \ln \left(\frac{R+z_{\mu}}{R-z_{\mu}}\right)=0
$$

das bisherige Ergebnis (A 14) weiter zu

$$
F(\alpha)=2 \pi i\left(f_{1}+f_{2}\right) .
$$

Mit diesem Resultat können Integrale vom Typ (A 5) geschlossen berechnet werden.

[10] K. Dräger, Z. Naturforsch. 32 a, 163 (1977).

[11] J. H. van Vleck, Phys. Rev. 74, 1168 (1948).

[12] R. Gerling, Dissertation, Universität Hamburg 1978.

[13] M. Griffel u. J. W. Stout, J. Amer. Chem. Soc. 72, $4351(1950)$

[14] P. W. Anderson u. P. R. Weiss, Rev. Mod. Phys. 25, 269 (1953).

[15] P. W. Anderson, J. Phys. Soc. Japan 9, 316 (1954).

[16] W. J. C. Grant, Phys. Rev. 135 A, 1265 (1964); Phys. Rev. 134 A, 1554 (1964); Phys. Rev. 134 A, 1564 (1964).

[17] E. Buluggiu, A. Dall'Oho u. V. Varacca, Phys. stat. sol. 32, 745 (1969).

[18] J. E. Gulley, D. Hone, D. J. Scalapino u. B. G. Silbernagel, Phys. Rev. B 1, 1020 (1970).

[19] L. V. Ahlfors, Complex Analysis, McGraw-Hill, New York, 1953. 